\title{
EFECTO DEL NIVEL DE PROTEÍNA CRUDA EN LA DIETA SOBRE EL CRECIMIENTO Y EXCRECIÓN DE NITRÓGENO AMONIACAL TOTAL DE Carassius auratus (Cyprinidae) Linnaeus (1758) BAJO CONDICIONES DE LABORATORIO
}

Fecha de recepción: 19 de noviembre de 2014 • Fecha de aceptación: 17 de diciembre de 2014

\section{EFFECT OF DIETARY CRUDE PROTEIN LEVEL ON GROWTH AND TOTAL AMMONIA NITROGEN EXCRETION OF Carassius auratus (Cyprinidae) Linnaeus (1758) UNDER LABORATORY CONDITIONS}

\author{
Edna R Riaño Castillo • Ana C Torres Mesa² • Hernán Hurtado Giraldo 3,5 Edwin Gómez Ramírez
}

\section{RESUMEN}

Para el diseño de los sistemas cerrados de recirculación (SCR) y sistemas acuapónicos (SA) es importante conocer la tasa de excreción de nitrógeno amoniacal total (NAT) de la especie cultivada. Carassius auratus es un ornamental muy cultivado, debido a su tolerancia a diferentes condiciones ambientales. En este estudio se determinó el efecto del nivel de proteína cruda en la dieta sobre el crecimiento y excreción de NAT de C. auratus. Se evaluaron tres niveles de proteína cruda (30\%, 40\% y 45\%) por un periodo de tres meses en acuarios de $40 \mathrm{~L}$ a una densidad de 10peces/acuario. La temperatura del agua se mantuvo en $24 \pm 1^{\circ} \mathrm{C}$ y pH: $6.5-7$. Los peces se alimentaron tres veces al día al $5 \%$ de la biomasa total. Semanalmente se llevó un control de parámetros fisicoquímicos: NAT, $\mathrm{NO}_{2} ; \mathrm{NO}_{3} ; \mathrm{GH}, \mathrm{KH}, \mathrm{pH}$, temperatura y oxígeno disuelto. Mensualmente se registró la tasa de excreción de NAT y el crecimiento (peso, longitud total y estándar). Al final del experimento se estimaron los parámetros productivos de la especie. Los resultados indican que los parámetros productivos fueron similares en los tres tratamientos a excepción de GP, TCA y CR ( $\mathrm{p}<0.05)$. El NAT excretado en C. auratus fue: 0.00075-0.0043 mgNAT/g de pez/día (30\%PC); 0.001-0.037 mgNAT/g de pez/día (40\%PC); 0.007-0.038 mgNAT/g de pez/día (45\%PC). La información obtenida es de gran utilidad para el diseño de SCR y SA, pues permite establecer el tamaño del biofiltro, realizar cálculos de balance de masas y estimar el $\mathrm{NO}_{3}{ }_{3}$, importante nutriente en el crecimiento de plantas.

Palabras clave: Sistemas de recirculación, sistemas acuapónicos, parámetros productivos y parámetros fisicoquímicos, goldfish.

1 Biólogo, Joven Investigador Grupo de Ictiología, Facultad de Ciencias Básicas. Universidad Militar Nueva Granada.

2 Biólogo, Asistente Investigador Grupo de Ictiología, Facultad de Ciencias Básicas. Universidad Militar Nueva Granada.

3 Docente, Director de Grupo de Ictiología, Facultad de Ciencias Básicas. Universidad Militar Nueva Granada.

4 Biólogo, Esp. MSc, Asistente Investigador Grupo de Ictiología, Facultad de Ciencias 5.Básicas. Universidad Militar Nueva Granada.

5 Autor para correspondencia: hernan.hurtado@unimilitar.edu.co. 


\section{ABSTRACT}

In order to design and improve closed recirculatory and aquaponic systems, it is important to know the total ammonia nitrogen (TAN) excretion rate of cultured fish. Carassius auratus is an ornamental species form East Asia and one of the most cultured due to its tolerance to different environmental conditions. The main goal of this work was to determine the effect of diet raw protein level on C. auratus growth and TAN excretion. Three raw protein levels were evaluated (30\%, $40 \%$ and $45 \%$ ) for three months at 10 fishes/40 I aquarium. Water temperature was kept at $24 \pm 1^{\circ} \mathrm{C}$ and $\mathrm{pH}$ at 6.5-7. Fishes were feed three times a day, at $5 \%$ of total biomass. NAT excretion rate and growth (body mass, total and standard lenght were taken monthly. Each week physicochemical parameters TAN, $\mathrm{NO}_{2}, \mathrm{NO}_{3}, \mathrm{GH}, \mathrm{KH}, \mathrm{pH}$, temperature and dissolved oxygen were obtained. Productive parameters were established at the end of the experiments. All fishes exhibited a similar productive parameter, excepted for GP. TCA amd CR $(p<0.05)$. NAT excretion rate shown by $C$. auratus were $0.00075-$ $0.0043 \mathrm{mgNAT} / \mathrm{g}$ of fish/day (30\%); 0.001-0.037 mgNAT/g of fish/day (40\%); 0.007-0.038 mgNAT/g of fish/day (45\%). This information is usefull for SCR and SA design, as it allows biofilter sizing, mass balance calculations, and $\mathrm{NO}_{3}$ - level estimation.

Key words: Recirculatory systems, aquaponic systems, productive parameters, physicochemical parameters, goldfish.

\section{INTRODUCCIÓN}

En el metabolismo de aminoácidos, el grupo amino es liberado (desaminado) y transferido a otra molécula para su eliminación o reutilización (Wilkie, 2002; Randall, 2011). Los grupo amino que no son reutilizados en la síntesis de aminoácidos, deben ser excretados para evitar su acumulación en el plasma sanguíneo y en los tejidos del organismo (Wilkie, 2002; Randall, 2011). La mayoría de peces teleósteos son amoniotélicos, puesto que el exceso de nitrógeno liberado durante la desaminación es excretado en forma de $\mathrm{NH}_{3}$ (principal producto excretado y producto final del catabolismo proteico). Los organismos amoniotélicos utilizan esta estrategia principalmente por dos factores: primero porque tiene un menor costo energético, puesto que no convierten el amonio a urea y ácido úrico (Altinok y Grizzle, 2004); y segundo porque el $\mathrm{NH}_{3}$ puede difundirse fácilmente a través de la membrana branquial, debido a su solubilidad en lípidos y a su falta de carga (Sinha et al., 2012).

En el agua, el amonio se encuentra de dos formas: la no ionizada denominada amoniaco $\left(\mathrm{NH}_{3}\right)$ y la ionizada denominada amonio $(\mathrm{NH} 4+$ ); la suma de estos dos compuestos se denomina como nitrógeno amoniacal total (NAT) (Wajsbrot et al., 1993; Randall y Tsui, 2002). La forma más tóxica es la forma no ionizada $\left(\mathrm{NH}_{3}\right)$, siendo un contaminante común en sistemas acuáticos (Yuxiang y Patrick, 2000; Wood, 2006). La acumulación de esta molécula en los sistemas acuícolas puede disminuir el crecimiento de la especie, debido a que causa cambios en la estructura de las branquias, interfiriendo en el proceso fisiológico de intercambio de iones; causa una mayor 
susceptibilidad a patógenos debido al daño de los tejidos epidérmicos y puede interferir en el metabolismo de oxígeno, ya que se une a la hemoglobina (heteroproteína encargada de transportar el oxigeno por el cuerpo), interfiriendo en la captura del oxigeno por parte de los eritrocitos, causando hipoxia y en ocasiones muerte del organismo (Wilkie, 1997; Wilkie, 2002; Sinha et al., 2012).

La cuantificación de la tasa de excreción de nitrógeno amoniacal total (NAT) es útil e importante en sistemas cerrados de recirculación (SCR), ya que permite estimar la carga máxima en biomasa/densidad en peces; el flujo de agua y el tamaño de los filtros biológicos para mantener las concentraciones de NAT ideal (Leung et al., 1999; Crear y Forteath, 2002). Además en sistemas acuapónicos permite definir la cantidad de compuestos nitrogenados producidos por los peces para estimar la cantidad de plantas que pueden ser mantenidas en los sistemas (Rakocy, 2007).

La tasa de excreción de NAT en teleósteos está influenciado por muchos factores: el nivel de proteína cruda (PC) en la dieta, la temperatura, el pH, el peso corporal, la calidad y digestibilidad de la dieta y la especie del pez (Ruchimat et al., 1997; Randall y Tsui, 2002; Webb y Gatlin, 2003; Merino, 2007; Timmons MB y Ebeling JM, 2007; Tulli et al., 2007; Singh et al., 2008; Nerici et al., 2012). En teleósteos, una de las fuentes principales de nitrógeno son los aminoácidos obtenidos de la digestión de proteínas; se ha demostrado que el nivel de proteína afecta la excreción de compuestos nitrogenados, mostrando una relación directamente proporcional (Chakraborty y Chakraborty, 1998; Randall y Tsui, 2002; Singh et al., 2008). Un incremento en el porcentaje de proteína cruda en la dieta eleva los niveles de aminoácidos presentes en los organismos y por lo tanto el catabolismo de las proteínas y la excreción de $\mathrm{NH}_{3}$ aumentan, causando una mayor concentración de nitrógeno amoniacal total (NAT) en el agua (Ballestrazzi et al., 1998; Wicks y Randall, 2002).
Carassius auratus (Goldfish) es una especie ornamental con gran impacto comercial y una de la más cultivadas, alcanzando una producción en el 2010 de 69 t en América (México y Estados Unidos), 167 t en Asia y 4815 t en Europa (FAO, 2012). Además ha sido un modelo excelente para estudios científicos (Gómez et al., 2006; Hanington et al., 2006; Komiyama et al., 2009), debido a que es un organismo capaz de tolerar diferentes condiciones ambientales. Por lo tanto, el objetivo de este estudio fue evaluar el efecto de tres niveles de proteína cruda (30, 40 y 45\%) en el crecimiento y excreción de NAT de Carassius auratus, con el fin de otorgar información básica para estudios posteriores orientados en la excreción de NAT y su importancia en sistemas cerrados de recirculación y sistemas acuapónicos.

\section{MATERIALES Y MÉTODOS}

El estudio se llevó a cabo en el Laboratorio de Fisiología Animal del Campus de la Universidad Militar Nueva Granada, Cajicá (Cundinamarca), Colombia (N 4 56' 29.67"; $O 74^{\circ} 00^{\prime} 54.50^{\prime \prime}$ ), durante un periodo de tres meses (Marzo- Junio del 2012). Se trabajó con 90 individuos de la especie Carassius auratus, con un peso promedio de $2.69 \pm 0.99 \mathrm{~g}-2.82 \pm 1.10 \mathrm{~g}$. Antes de iniciar el experimento los individuos fueron mantenidos en cuarentena, durante 15 días, en sistemas cerrados de recirculación de 250L, alimentados con concentrado comercial Tilapia ${ }^{\circledR}$ al $30 \%$ de proteína cruda (PC). Durante este tiempo fue controlada la calidad del agua, por medio de la medición de parámetros fisicoquímicos (NAT, nitrito $\left(\mathrm{NO}_{2}{ }^{-}\right)$, nitrato $\left(\mathrm{NO}_{3}{ }^{-}\right)$, Oxígeno disuelto (OD), dureza general $(\mathrm{GH})$, dureza de carbonatos $(\mathrm{KH})$, temperatura $\left({ }^{\circ} \mathrm{C}\right)$ y $\left.\mathrm{pH}\right)$.

\section{Manejo de material biológico}

De igual manera, antes de iniciar el experimento se realizó un control de calidad de agua en cada unidad experimental, donde cada acuario se adaptó a las 
siguientes condiciones fisicoquímicas: Temperatura $24 \pm 1{ }^{\circ} \mathrm{C}$; pH 6.5-7.0; Oxigeno disuelto (OD) > 4ppm y $\mathrm{NAT} \leq 0.50 \mathrm{mg} / \mathrm{Lppm}$. Estas condiciones son reportadas como los rangos óptimos para C. auratus (Dinaria-FAO, 2008) y fueron controladas durante todo el experimento.

Terminada esta adaptación se sembraron 10 peces/acuario (Marshall, 1995) en cada unidad experimental y durante la primera semana los peces fueron alimentados con el nivel de proteína cruda correspondiente al tratamiento (T1: 30\%; T2: 40\% y T3: 45\%). Pasada esta semana, después de la adaptación de los organismos a las condiciones experimentales se inicia con el experimento.

\section{Diseño experimental}

Se realizó un diseño experimental completamente aleatorizado (DCA), donde se evaluaron 3 tratamientos (T1, T2 y T3) cada uno con 3 repeticiones, para un total de 9 acuarios con un volumen de agua de 40L/acuario.

Tres niveles de proteína cruda (PC) fueron evaluados: $30 \%$, 40\% y 45\%. Se empleó concentrado comercial de Tilapia ${ }^{\circledR}$ para las unidades experimentales con 30\% PC y concentrado comercial Truchina ${ }^{\circledR}$ para las unidades experimentales de $40 \%$ y $45 \%$ PC. Los individuos fueron alimentados tres veces al día (8:00h, 12:00h y 16:00h) con el 5\% de la biomasa total.

Los montajes contaron con aireación constante por medio de una turbina (GF 250-280 Watt); se introdujeron dos difusores por unidad experimental; se incorporó un termostato/acuario de $150 \mathrm{~W}$ para mantener la temperatura a $24 \pm 1^{\circ} \mathrm{C}$ y un sistema de biofiltración/acuario para mantener controlado la concentración de NAT y no permitir que haya concentraciones tóxicas que puedan afectar la salud y el crecimiento de los peces.

\section{Parámetros fisicoquímicos}

Diariamente se registró $\mathrm{pH}$ y temperatura $\left({ }^{\circ} \mathrm{C}\right)$ con un potenciómetro con sensor de temperatura marca SCHOTT ${ }^{\circledR}$. Semanalmente se midió el oxígeno disuelto (OD) con el oxímetro modelo DO-700 de Extech Instruments ${ }^{\circledR}$ con ajuste de compensación a la altura; $\mathrm{GH}$ y $\mathrm{KH}$ por medio de kits comerciales (TETRA Test Laborett $\left.{ }^{\circledR}\right)$; NAT, nitrito $\left(\mathrm{NO}_{2}{ }^{-}\right)$y nitrato $\left(\mathrm{NO}_{3}{ }^{-}\right)$con el colorímetro Spectroquant ${ }^{\circledR}$ Multy y con kits de alta sensibilidad MERCK ${ }^{\circledR}$.

\section{Parámetros de Crecimiento.}

Para estimar el crecimiento de C. auratus se realizó al inicio del experimento un registro de peso, longitud total (LT) y longitud estándar (LS) de los 10 individuos/acuario; posteriormente se llevó a cabo un seguimiento de estos valores por medio de la medición de 5 individuos/acuario de cada tratamiento, cada tres semanas, después de tomar el registro de la tasa de excreción de NAT, con el fin de no estresar a los individuos y consecuentemente alterar los resultados. El registró del peso se realizó a través de una balanza (OHAUS MODEL Adventurer Pro AV 2101), y el de la longitud total y estándar con un calibrador (Vernier Caliper; medición 0-150mm). Adicionalmente se calcularon parámetros productivos (Tabla 1) al finalizar el estudio.

\section{Medición de excreción de nitrógeno amoniacal total (NAT)}

La medición de NAT se realizó de la siguiente manera: antes de alimentar a los peces con la primera ración de comida se midió el NAT inicial en cada unidad experimental; luego los peces fueron alimentados a las 8:00 am, 12:00 y 16:00 pm con el nivel de proteína correspondiente al tratamiento y pasada las 24 horas (8:00 am del otro día) se midió el NAT final/acuario; este procedimiento se realizó durante tres días. Posteriormente se llevo a cabo un seguimiento de estos parámetros cada tres semanas por un periodo de tres meses (94 días). Se realizó medicines a los 10, 35, 64 y 94 días, para un total de cuatro muestreos. El primer muestreo se realizó a los 
Tabla 1. Parámetros productivos para la evaluación del crecimiento de C. auratus.

\begin{tabular}{|c|c|c|}
\hline Parámetro productivo & Fórmula & Referencia \\
\hline Ganancia de peso & $\mathrm{GP}=$ peso final $(\mathrm{g})$ - peso inicial $(\mathrm{g})$ & Mercado et al., 2006 \\
\hline $\begin{array}{c}\text { Tasa de } \\
\text { Crecimiento absoluto }\end{array}$ & $\begin{aligned} \mathrm{TCA} & =[(\text { peso final }(\mathrm{g}) \text {-peso incial }(\mathrm{g})) / \\
& (\text { tiempo final }- \text { tiempo inicial })]\end{aligned}$ & Salazar y Ocampo, 2002 \\
\hline Crecimiento relativo & $\begin{array}{c}\text { CR }=100 \times[(\text { peso final }(g)-\text { peso inicial }(g)) / \\
\text { peso inial }(\mathrm{g})]\end{array}$ & Mercado et al., 2006 \\
\hline Factor de condición $\mathrm{K}$ & $\mathrm{K}=\left(\right.$ peso / Longitud $\left.{ }^{3}\right) \times 100$ & Mercado et al., 2006 \\
\hline $\begin{array}{l}\text { Factor de } \\
\text { conversión alimenticia }\end{array}$ & $\begin{array}{l}\text { FCA = alimento consumido }(\mathrm{g}) / \\
\text { incremento de peso }(\mathrm{g})\end{array}$ & Salazar y Ocampo, 2002 \\
\hline $\begin{array}{l}\text { Tasa de } \\
\text { eficiencia proteica }\end{array}$ & $\mathrm{TEP}=$ ganancia de peso $(\mathrm{g}) /$ proteína consumida $(\mathrm{g})$ & Mercado et al., 2006 \\
\hline $\begin{array}{l}\text { Tasa de eficiencia } \\
\quad \text { alimenticia }\end{array}$ & $\begin{array}{l}\text { TEA = Incremento de peso }(\mathrm{g}) / \\
\text { alimento consumido }(\mathrm{g})\end{array}$ & Mercado et al., 2006 \\
\hline Supervivencia & $\begin{array}{c}S=100 \times(\text { número de peces final } / \\
\text { número de peces inicial) }\end{array}$ & $\begin{array}{l}\text { Salazar y Ocampo, 2002; } \\
\text { Mercado et al., } 2006\end{array}$ \\
\hline
\end{tabular}

10 días después de la adaptación de los organismos a las condiciones experimentales.

Al momento de medir el NAT inicial en cada unidad experimental se realizo un correctivo, que consistía en hacer un recambio del $50 \%$ de agua a los acuarios que presentaban una concentración de NAT >0.50 mg/L. Además antes de cada tiempo de muestreo se retiraron los sistemas de biofiltración, con el fin de evitar el proceso de nitrificación (convertir el NAT a nitrito y el nitrito a nitrato) y no alterar los resultados del experimento.

Con los valores obtenidos de NAT se determinó la tasa de excreción de NAT/individuo y la tasa de excreción por gramo de pez / hora, la cual fueron calculadas con las siguientes fórmulas:

\section{Formula 1:}

Tasa de excreción de NAT/Individuo $=[\Sigma((N A T$ final $_{\text {dia } 1-}$ NAT inicial $\left.{ }_{\text {día } 1}\right)+\left(\right.$ NAT final $_{\text {día }}$ - NAT inicial $\left.{ }_{\text {día } 2}\right)+$ (NAT final día NAT inicial ${ }_{\text {día }}$ )) / Número de individuos]
Formula 2:

Tasa de excreción de NAT por gramo de pez / hora $=$

$\left[\Sigma\left(\left(\right.\right.\right.$ NAT final dia1- NAT inicial $\left.{ }_{\text {día }}\right)+\left(\right.$ NAT final día $^{-}$ NAT inicial día $)+\left(\right.$ NAT final $_{\text {dia3 }^{-}}$NAT inicial $\left.\left.{ }_{\text {dia } 3}\right)\right)$ / Biomasa total] / 72 horas.

Donde, $\Sigma$ (NAT $_{\text {final }}$ - NAT ${ }_{\text {inicial }}$ ) es la suma del NAT final (concentración NAT (mg/L) al final del período de medición) con NAT inicial (concentración de NAT inicial (mg/L) al comienzo del período de medición) durante los tres días (72h) y el Número de individuos, es el número total de individuos o la sobrevivencia en el tiempo de muestro.

\section{Análisis estadístico}

Las variables respuesta (tasa de excreción de NAT, peso, longitud total y estándar de los individuos de C. auratus) fueron evaluados con la prueba 
de normalidad Shapiro. Se realizó homogeneidad de varianza y análisis de varianza, implementando la prueba ANOVA y Tukey, individual para cada variable, al nivel de significancia del $5 \%(P<0.05$, error tipo I), con el fin de evaluar las variables respuesta y determinar si hubo diferencias entre los tratamientos. El efecto de peso y el nivel de proteína cruda en la excreción de NAT fueron evaluados utilizando el análisis de regresión múltiple. Para ello, se empleó el paquete estadístico $R$ versión 2.12.2, de libre distribución en internet (http://r-project.softonic.com/).

Adicionalmente con los datos de peso y NAT se calculó el promedio \pm error estándar y se realizaron gráficas en función del tiempo. Del mismo modo, con los datos de peso, longitud total y estándar se calculó el promedio \pm error estándar y se realizó gráficas para determinar el comportamiento del crecimiento a lo largo del experimento.

\section{RESULTADOS Y DISCUSIÓN}

Efecto del nivel de proteína cruda en la dieta sobre el crecimiento de $C$. auratus

Durante los primeros 68 días la prueba ANOVA no arrojó diferencias significativas ( $p>0.05)$ entre todos los tratamientos, mostrando un crecimiento similar en peso, longitud total (LT) y longitud estándar (LS). Sin embargo en el último muestreo (94 días) el tratamiento 3 (T3), alimentado con proteína cruda (PC) del $45 \%$ fue significativamente mayor $(p<0.05)$ en peso sobre el tratamiento 1 (T1), pero similar con el tratamiento 2(T2); mientras que el crecimiento en LS mostró un aumento significativo de $p<0.05$ sobre el T1 y T2, pero el T1 y T2 fueron similares entre sí y en LT no se presentaron diferencias significativas entre los tratamientos (Fig.1).

En acuicultura, la proteína es el componente más estudiado para mejorar el desempeño y maximizar el crecimiento de los organismos (Bandyopadhyay et al., 2005; Abdo et al., 2010). Yang et al. (2002) trabajo con juveniles (2.72 g) de perca plateada (Bidyanus
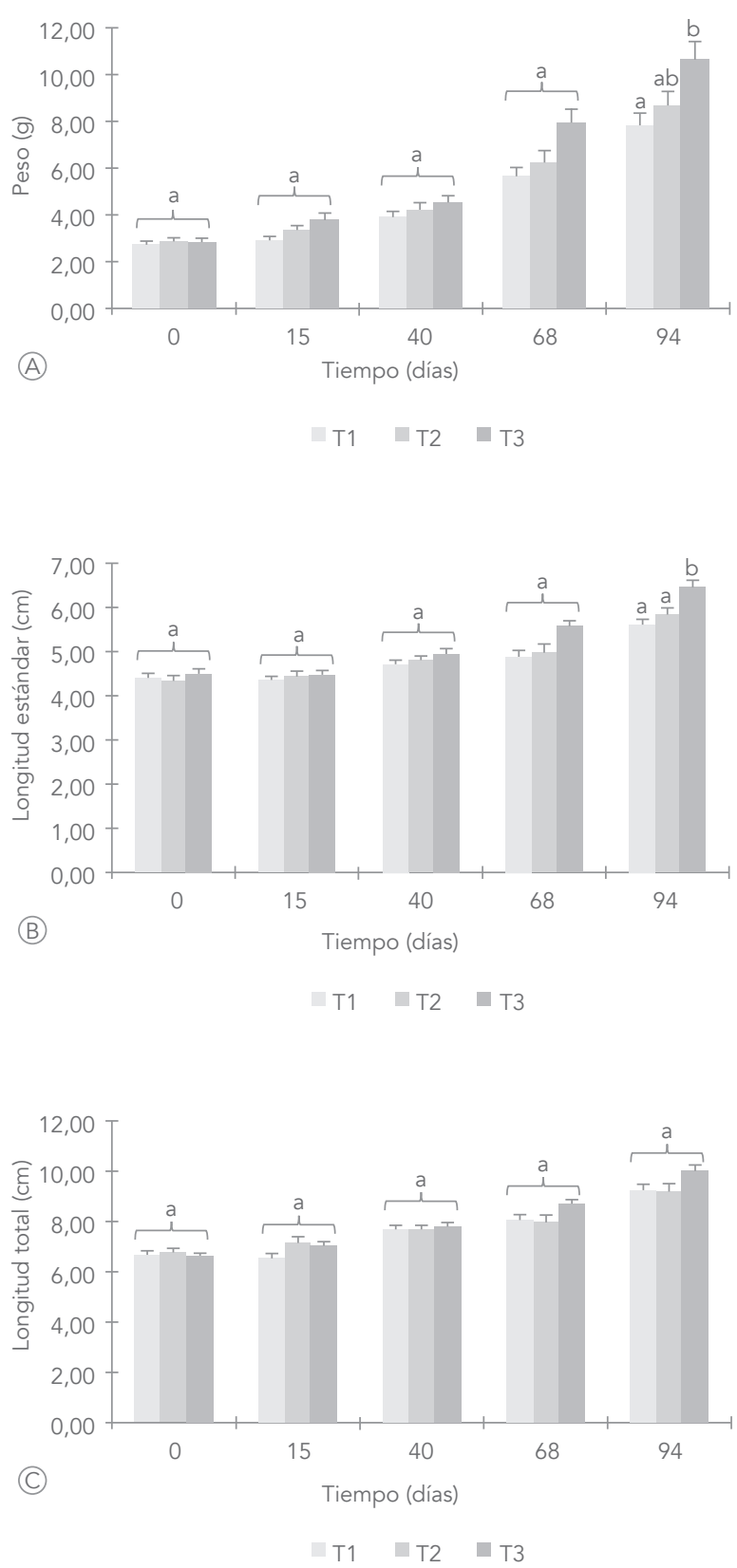

Figura 1. Efecto de tres niveles de proteína en el crecimiento de C. auratus. Los valores representan el valor promedio \pm error estándar. Las letras diferentes representan diferencias significativas entre tratamientos $(p<0.05)$. A. Crecimiento en peso; B. Crecimiento en longitud estándar; C. Crecimiento en longitud total. T1: 30\% PC; T2: 40\% PC; T3: 45\% PC. 
bidyanus), una especie de agua dulce y Singh et al. (2008) trabajo con una especie de la familia Cyprinidae, comúnmente llamada carpa India (Cirrhinus cirrhosus), en el cual observaron que el crecimiento de los peces aumentaba con el incremento del nivel de proteína cruda en la dieta. En este trabajo, mostramos como la concentración de proteína cruda en la dieta de C. auratus incrementa su crecimiento como lo muestran las variables de peso y longitud estándar en T3 (Fig. 1).

En cuanto a los parámetros productivos, el análisis estadístico mostró diferencias significativas $(p<0.05)$ entre T1 y T3 para GP, TCA y CR y diferencias significativas entre T2 y T3 para CR, evidenciando un mejor desempeño en el tratamiento 3, con alto nivel de PC: $45 \%$ (Tabla 2). Al comparar los resultados con trabajos previos en la misma especie como los reportados por Martínez et al. (2011) el desempeño productivo fue considerablemente mayor. Martínez et al. (2011) em-

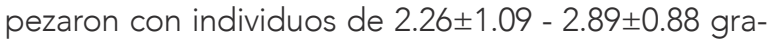
mos y durante cuatro meses y con el nivel de proteína de $45 \%$ alcanzaron una GP de 0.7- $1 \mathrm{~g}$ y un peso final de $4.23 \pm 0.43$ - 4.69 $\pm 0.44 \mathrm{~g}$; mientras en este trabajo se inició con un peso de $2.69 \pm 0.99$ - 2.82 $\pm 1.10 \mathrm{~g}$ y en un tiempo menor (tres meses) la GP fue de $5.5-7.5 \mathrm{~g}$ y el peso final de $7.79 \pm 0.54-10.61 \pm 0.80 \mathrm{~g}$. De igual manera ocurrió para los otros parámetros productivos (TCA, CR, FCA y TEA). Sin embargo, Martínez et al. (2011) trabajaron con una temperatura promedio de $17^{\circ} \mathrm{C}$ y en este estudio la temperatura promedio fue de $24 \pm 1^{\circ} \mathrm{C}$ lo que podría explicar las diferencias encontradas.

Por otro lado, los datos de FCA, TEP y TEA no presentaron diferencias significativas $(p>0.05)$ entre los tratamientos (Tabla 2). No obstante, se observó que al incrementar el nivel de proteína cruda disminuyó el factor de condición alimenticia (FCA) y aumentó la tasa de eficiencia alimenticia (TEA), presentando el T3 ali-

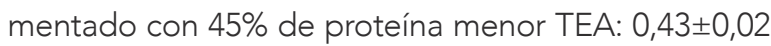
y mejor FCA: 2.35 $\pm 0,09$ entre los tratamientos (Tabla 2); similar a lo obtenido por Mohammad (2009) que trabajó con Oreochromis niloticus (tilapia), una especie omnívora con tendencia herbívora. Para la acuicultura es recomendable obtener este comportamiento, donde la dieta que presenta mejor desempeño es la que obtenga alta TEA y bajo FCA (Corraze y Kaushik, 2007; Ilmo, 2009), pues TEA indica la asimilación del alimento por el pez para el crecimiento (Iregui et al., 2002) y FCA indica el alimento que hay que suministrar para cada unidad de peso ó biomasa. Es decir bajos valores significan que hay menor consumo de alimento por el pez para subir un gramo o kilogramo

Tabla 2. Parámetros productivos del crecimiento de C. auratus. Los valores representan el valor promedio \pm desviación estándar. Las letras diferentes representan diferencias significativas entre tratamientos ( $p<0.05)$. T1: $30 \%$ PC; T2: 40\% PC; T3: $45 \%$ PC.--

\begin{tabular}{cccc}
\hline TRATAMIENTO & T1 & T2 & T3 \\
\hline Supervivencia & $73,3 \pm 3,03 \mathrm{a}$ & $70 \pm 3,67 \mathrm{a}$ & $86,7 \pm 1,53 \mathrm{a}$ \\
\hline GP & $5,07 \pm 0,55 \mathrm{a}$ & $5,78 \pm 0,90 \mathrm{ab}$ & $7,74 \pm 1,32 \mathrm{~b}$ \\
\hline TCA & $0,05 \pm 0,01 \mathrm{a}$ & $0,06 \pm 0,01 \mathrm{ab}$ & $0,08 \pm 0,01 \mathrm{~b}$ \\
\hline CR & $188,90 \pm 19,26 \mathrm{a}$ & $204,60 \pm 24,66 \mathrm{a}$ & $279,91 \pm 25,96 \mathrm{~b}$ \\
\hline K & $4,48 \pm 0,47 \mathrm{a}$ & $4,51 \pm 0,61 \mathrm{a}$ & $3,93 \pm 0,28 \mathrm{a}$ \\
\hline FCA & $2,73 \pm 0,23 \mathrm{a}$ & $2,61 \pm 0,12 \mathrm{a}$ & $2,35 \pm 0,09 \mathrm{a}$ \\
\hline TEP & $0,17 \pm 0,02 \mathrm{a}$ & $0,14 \pm 0,02 \mathrm{a}$ & $0,17 \pm 0,03 \mathrm{a}$ \\
\hline TEA & $0,37 \pm 0,03 \mathrm{a}$ & $0,38 \pm 0,02 \mathrm{a}$ & $0,43 \pm 0,02 \mathrm{a}$ \\
\hline
\end{tabular}


de biomasa, siendo económicamente más rentable (Iregui et al., 2002).

En este orden de ideas, se puede decir que el nivel de proteína influye significativamente en el crecimiento de C. auratus. Una explicación a esto se debe a que la proteína es un componente de gran importancia en la dieta de los peces, puesto que el crecimiento es en gran parte consecuencia de la síntesis proteica, resultado de dos procesos metabólicos (el catabolismo y anabolismo), donde la proteína que constituye la dieta de los peces es digerida y los aminoácidos son usados para la síntesis de nuevas proteínas, para la formación y regeneración de tejidos (como músculo, piel, células sanguíneas, enzimas, entre otros), para la producción de energía y para funciones metabólicas y reguladoras. (Corraze y Kaushik, 2007; Ilmo, 2009; Santosh y Tibbetts, 2009).

De igual manera, se considera que el alto nivel de proteína cruda (40 y 45\%) en la dieta fue esencial para el crecimiento de C. auratus, principalmente porque los ejemplares eran juveniles, de pequeña taIla $(2,70 \pm 1,10 \mathrm{~g})$, los cuales presentaban una alta tasa metabólica en comparación con otros organismos de mayor tamaño; esto debido a la relación área/volumen (Ilmo, 2009), ya que los peces y en general los organismos más pequeños tiene comparativamente mayor área expuesta que organismos de mayor tamaño, por lo cual, esto hace que consuman mayor energía y requieran mas proteína para satisfacer las necesidades metabólicas (Richard, 1980; Ilmo, 2009). Lo anterior es apoyado por Bandyopadhyay et al. (2005), donde reporta que las tallas juveniles de $C$. auratus requieren alta cantidad de proteína para un crecimiento óptimo, presentando un mejor desempeño al ser alimentados con dietas de proteína cruda del $40 \%$ y $45 \%$.

Efecto del nivel de proteína cruda en la dieta sobre la tasa de excreción de nitrógeno amoniacal total (NAT) en C. auratus.

La relación entre el porcentaje y la calidad de PC en la dieta y la excreción de los productos nitrogenados, son factores que pueden actuar como elementos de estrés para los organismos acuáticos (Randall y Tsui, 2002; Guan et al., 2010). Una de las fuentes de nitrógeno en el sistema animal son los aminoácidos obtenidos de la digestión de proteínas. Una vez absorbidos estos aminoácidos pueden ser usados para construir nuevas proteínas o pueden ser desaminados y los residuos ser utilizados para formar otros compuestos (amoniaco, urea y ácido úrico) (Engin y Carter, 2001; Wilkie, 2002; Merino et al., 2007).

El nivel de proteína afecta directamente y de manera proporcional la excreción de NAT, lo cual ha sido descrito por varios autores en otras especies de peces (Engin y Carter, 2001; Yang et al., 2002; Guo et al., 2012). En el estudio se observó que el T3, tratamiento con mayor nivel de proteína cruda (45\%) presentó la mayor tasa de excreción de NAT entre los tratamientos, exhibiendo a los 10 días diferencias significativas de $\mathrm{p}<0.05$ frente al tratamiento 1 y a los 94 días frente al tratamiento 2 y 1 (Tabla 3); por otra parte, se observó que el tratamiento 2 presentó a los 10 días diferencias significativas de $p<0.05$ frente al tratamiento 1 (Tabla 3) y presentó una excreción de NAT total significativamente mayor a los 10 días, en comparación a lo excretado en los días 35, 64 y 94 del experimento; mientras que el tratamiento 1 y 3 no presentaron diferencias significativas ( $p>0.05$ ) a lo largo del tiempo ( Fig. 2).

El comportamiento de estos datos refleja que el nivel de proteína en la dieta no es el único factor que afecta la tasa de excreción de NAT, también pudo haber otros factores que influyeron en la excreción como el tamaño y peso corporal de los individuos, pues al inicio del experimento donde se presentó una excreción significativamente mayor en los tratamientos de alta proteína (40 y 45\%) los individuos presentaban tallas pequeñas. De igual manera se observó una disminución (en relación al peso corporal) de excreción de NAT al aumentar la biomasa o peso corporal (Fig. 2 y Tabla 3); similar a lo obtenido por varios autores (Leung et al., 1999; Zhang et al., 2004; 

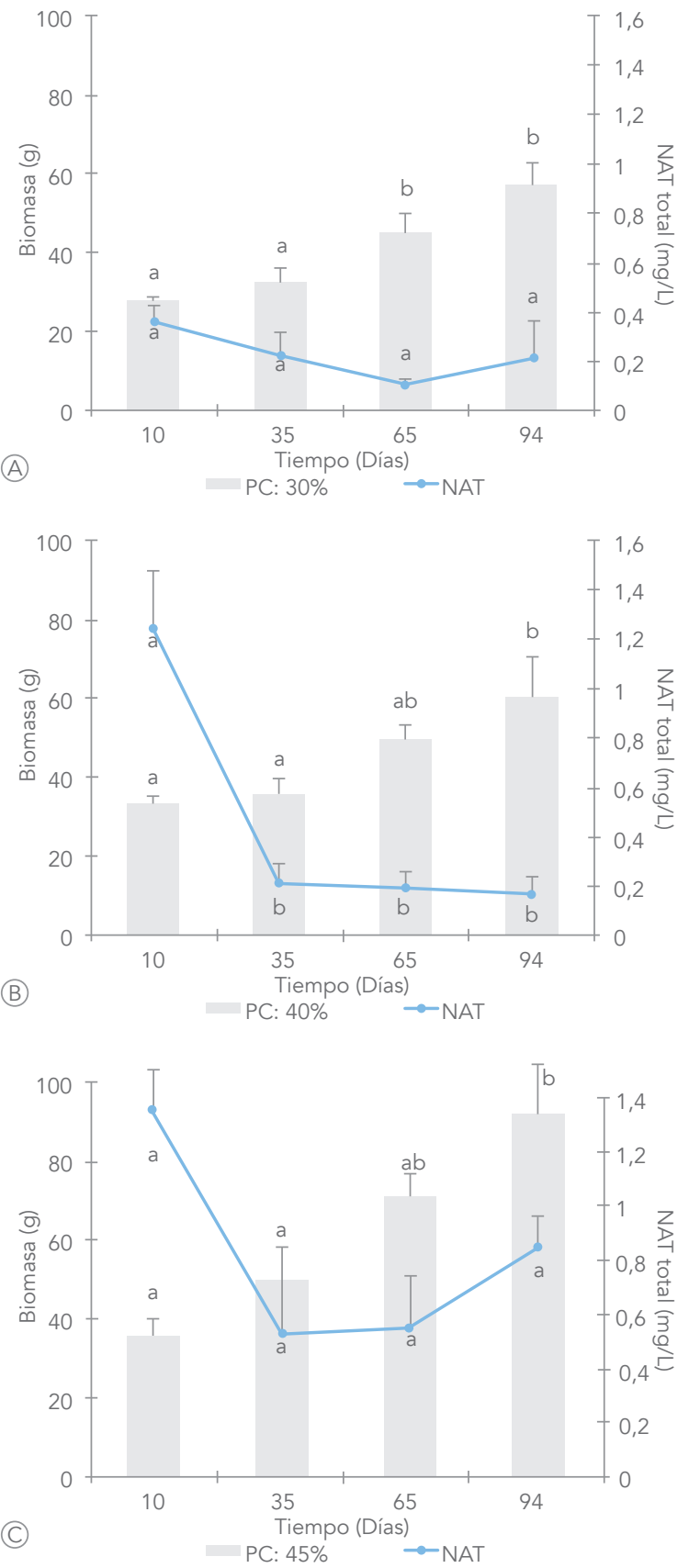

Figura 2 . Efecto de la biomasa y el nivel de proteína cruda $30 \%$ $40 \%$ y $45 \%$ ) sobre la excreción total de NAT en C. auratus. Los valores representan el valor promedio \pm error estándar. Las letras diferentes representan diferencias significativas entre el tiempo $(\mathrm{p}<0.05)$. A. Tratamiento 1 alimentado con el 30\% de PC: B. Tratamiento 2 alimentado con el $40 \%$ de PC; C. Tratamiento 3 alimentado con el $45 \%$ de PC.
Merino et al., 2007; Valbuena y Vásquez, 2011). Lo anterior demuestra que el tamaño y/o peso corporal de los organismos tiene una gran incidencia en el metabolismo de los peces, donde los peces de pequeña talla tienen una mayor área expuesta y su tasa metabólica es mayor frente a los peces de menor talla o peso corporal. Así mismo los peces de menor talla corporal consumen una mayor cantidad de alimento para suplir la energía requerida para su actividad metabólica, lo que se verá reflejado en la tasa de excreción de NAT (Ilmo, 2009). Un ejemplo de ello es el estudio realizado por Liu et al., (2009) que evaluó dos tamaños corporales (104 g y $173 \mathrm{~g}$ ) en palmetto bass (comúnmente llamada lobina), una especie hibrida, procedente del cruce de Morone sexatilis (una especie omnívora) con Morone chrysops (una especie carnívora), donde encontraron una mayor tasa de excreción de NAT en los peces de menor talla $(17.34 \pm 0.62$ mgNAT / $\mathrm{Kg} / \mathrm{h}$ ), en comparación a los peces de mayor talla corporal $(11.88 \pm 0.45 \mathrm{mgNAT} / \mathrm{Kg} / \mathrm{h})$.

En general para T3 los datos reflejan una tasa de excreción mayor, en comparación con la tasa de excreción encontrada en T2 y T1. Esto puede ser una consecuencia del aumento de proteína en la dieta que genera una mayor síntesis de proteínas aumentando la tasa catabólica de los aminoácidos lo que se refleja finalmente en un aumento de producción y excreción de NAT (Lim et al., 2001; Sinha et al., 2012). Lo anterior es apoyado por el análisis de regresión múltiple $\left(R^{2}=0.3627 ; F=10.62 ; P=1.248 e-05\right),(n=36)$, pues entre las variables de nivel de proteína cruda y peso corporal el análisis nos demuestra que la excreción de NAT en C. auratus está más relacionada o más influenciada por la proteína cruda en la dieta.

Los parámetros fisicoquímicos determinan el estado de salud de los peces afectando el crecimiento, la reproducción y sobrevivencia de los mismos (Timmons y Ebeling, 2007). Razón por la cual, su continuo análisis y seguimiento es de gran importancia para un adecuado crecimiento de los individuos (Timmons y 
Tabla 3. Efecto del nivel de proteína cruda (PC) y el peso corporal en la tasa de excreción de nitrógeno amoniacal total (NAT). Los valores representan el valor promedio \pm error estándar. Las letras diferentes representan diferencias significativas $(p<0.05)$ entre tratamientos (filas). TRAT: tratamiento. T1: 30\% PC; T2: 40\% PC; T3: 45\% PC.

\begin{tabular}{|c|c|c|c|c|c|c|}
\hline Días & TRAT & Peso (g) & mg NAT/ind & $\begin{array}{c}\text { mg NAT/g de } \\
\text { pez/72h }\end{array}$ & $\begin{array}{c}\text { mg NAT/g de } \\
\text { pez/día }\end{array}$ & $\mu g$ NAT/g/h \\
\hline \multirow{3}{*}{10} & $\mathrm{~T} 1$ & $2.87 \pm 0.06 \mathrm{a}$ & $0.037 \pm 0.006 \mathrm{a}$ & $0.013 \pm 0.002 \mathrm{a}$ & $0.0043 \pm 0.0013 a$ & $0.18 \pm 0.057 \mathrm{a}$ \\
\hline & $\mathrm{T} 2$ & $3.32 \pm 0.24 a$ & $0.125 \pm 0.023 b$ & $0.037 \pm 0.004 b$ & $0.012 \pm 0.0027 b$ & $0.51 \pm 0.11 b$ \\
\hline & T3 & $3.74 \pm 0.059 \mathrm{a}$ & $0.140 \pm 0.019 b$ & $0.038 \pm 0.001 b$ & $0.012 \pm 0.0010 b$ & $0.52 \pm 0.04 b$ \\
\hline \multirow{3}{*}{35} & $\mathrm{~T} 1$ & $3.87 \pm 0.32 \mathrm{a}$ & $0.028 \pm 0.011 \mathrm{a}$ & $0.007 \pm 0.003 a$ & $0.0023 \pm 0.0018 \mathrm{a}$ & $0.09 \pm 0.07 a$ \\
\hline & $\mathrm{T} 2$ & $4.18 \pm 0.62 \mathrm{a}$ & $0.024 \pm 0.008 \mathrm{a}$ & $0.007 \pm 0.002 \mathrm{a}$ & $0.0023 \pm 0.0012 \mathrm{a}$ & $0.09 \pm 0.05 a$ \\
\hline & T3 & $5.27 \pm 0.75 a$ & $0.056 \pm 0.002 a$ & $0.011 \pm 0.004 a$ & $0.0036 \pm 0.0028 a$ & $0.15 \pm 0.11 \mathrm{a}$ \\
\hline \multirow{3}{*}{64} & T1 & $5.60 \pm 0.64 a$ & $0.013 \pm 0.003 a$ & $0.002 \pm 0.0002 \mathrm{a}$ & $0.00075 \pm 0.00015 \mathrm{a}$ & $0.03 \pm 0.006 a$ \\
\hline & $\mathrm{T} 2$ & $6.2 \pm 0.49 a$ & $0.024 \pm 0.008 a$ & $0.004 \pm 0.001 \mathrm{a}$ & $0.0013 \pm 0.0008 a$ & $0.05 \pm 0.03 a$ \\
\hline & T3 & $7.91 \pm 0.61 \mathrm{a}$ & $0.060 \pm 0.020 \mathrm{a}$ & $0.007 \pm 0.002 \mathrm{a}$ & $0.0024 \pm 0.001 \mathrm{a}$ & $0.10 \pm 0.04 a$ \\
\hline \multirow{3}{*}{94} & $\mathrm{~T} 1$ & $7.76 \pm 0.45 a$ & $0.027 \pm 0.019 a$ & $0.003 \pm 0.002 \mathrm{a}$ & $0.0011 \pm 0.0012 \mathrm{a}$ & $0.04 \pm 0.05 a$ \\
\hline & $\mathrm{T} 2$ & $8.6 \pm 0.60 \mathrm{a}$ & $0.027 \pm 0.012 \mathrm{a}$ & $0.001 \pm 0.001 \mathrm{a}$ & $0.0011 \pm 0.0008 \mathrm{a}$ & $0.04 \pm 0.03 a$ \\
\hline & T3 & $10.55 \pm 1.15 b$ & $0.098 \pm 0.015 b$ & $0.010 \pm 0.001 \mathrm{~b}$ & $0.0031 \pm 0.0011 b$ & $0.13 \pm 0.04 b$ \\
\hline
\end{tabular}

Ebeling, 2007). En el presente estudio, los parámetros fisicoquímicos (Tabla 4) se mantuvieron dentro de los rangos reportados como adecuados para $C$. auratus (Dinara-FAO, 2008), por lo cual, se considera que no afectaron negativamente el crecimiento y la excreción de de NAT en C. auratus.

Por otra parte, este trabajo presenta resultados que pueden ser aplicados directamente en sistemas de recirculación (SCR) y sistemas acuapónicos, pues a partir de ellos se puede establecer la capacidad de carga de los sistemas; estimar el número de plantas que pueden ser mantenidas en los sistemas acuapónicos y dimensionar el tamaño del biofiltro para mantener las concentraciones ideales de NAT (Crear BJ y Forteath, 2002; Timmons y Ebeling, 2007). El grupo de Ictiología de la Universidad Militar Nueva
Granada comúnmente implementa biofiltros similares al "trickling biofilter", que según Timmons y Ebeling (2007) para un medio con área de 100-300 m²/ $\mathrm{m}^{3}$ tiene una tasa de conversión de NAT de 0.2-1.0 $\mathrm{g} / \mathrm{m}^{2} /$ día a una temperatura de $15-20^{\circ} \mathrm{C}$. Tomando la menor tasa de conversión de NAT $\left(0.2 \mathrm{~g} / \mathrm{m}^{2} /\right.$ día $)$ y conociendo que individuos de $C$. auratus con peso promedio de $10.55 \pm 1.15 \mathrm{~g}$ excretan $0.0031 \mathrm{mg} / \mathrm{L}$ NAT/g de pez/día (T3:45\% PC; Tabla 3), se puede estimar el área de biofiltración para un sistema de recirculación de $2.500 \mathrm{~L}$, con una biomasa total de 30.000 $\mathrm{g}$ con individuos de peso promedio de $10.55 \pm 1.15 \mathrm{~g}$. A partir de estos datos se calculará el área de biofitración usando la siguiente ecuación (Losordo y Hobbs, 2000; Crear y Forteath, 2002): SSA: tasa de excreción de NAT / tasa de nitrificación. 
Tabla 4. Parámetros fisicoquímicos. Los valores representan el valor promedio \pm desviación estándar. SEM: Semana; TRAT: Tratamiento; NO ${ }_{2}:$ Nitrito; $\mathrm{NO}_{3}$ : Nitrato; OD: Oxígeno Disuelto; $\mathrm{GH}$ : Dureza general; $\mathrm{KH}$ : Dureza de carbonato.

\begin{tabular}{|c|c|c|c|c|c|c|c|}
\hline SEM & TRAT & NAT (mg/L) & $\mathrm{NO}_{2}^{-}(\mathrm{mg} / \mathrm{L})$ & $\mathrm{NO}_{3}^{-}(\mathrm{mg} / \mathrm{L})$ & OD (mg/L) & $\mathrm{GH}(\mathrm{mg} / \mathrm{L})$ & $\mathrm{KH}(\mathrm{mg} / \mathrm{L})$ \\
\hline \multirow{3}{*}{1} & $\mathrm{~T} 1$ & $0.50 \pm 0.01$ & $0.30 \pm 0.01$ & $12.5 \pm 0.01$ & $4.9 \pm 0.36$ & $11.86 \pm 10.27$ & $5.93 \pm 10.27$ \\
\hline & $\mathrm{T} 2$ & $0.83 \pm 0.01$ & $0.30 \pm 0.01$ & $12.5 \pm 0.01$ & $5.16 \pm 0.15$ & $5.93 \pm 10.27$ & 0 \\
\hline & T3 & $0.88 \pm 0.01$ & $0.30 \pm 0.01$ & $12.5 \pm 0.01$ & $4.5 \pm 0.25$ & $5.93 \pm 10.27$ & 0 \\
\hline \multirow{3}{*}{2} & $\mathrm{~T} 1$ & $1.23 \pm 0.09$ & $0.03 \pm 0.02$ & $12.5 \pm 0.01$ & $5.0 \pm 1.30$ & $17,8 \pm 0,1$ & $11.86 \pm 10.2$ \\
\hline & $\mathrm{T} 2$ & $1.72 \pm 0.13$ & $0.07 \pm 0.05$ & $12.5 \pm 0.01$ & $5.4 \pm 0.36$ & $17,8 \pm 0,1$ & $11,86 \pm 10.27$ \\
\hline & T3 & $2.16 \pm 0.25$ & $0.08 \pm 0.07$ & $16.6 \pm 7.20$ & $4.5 \pm 0.32$ & $17,8 \pm 0,1$ & $17,8 \pm 0.1$ \\
\hline \multirow{3}{*}{3} & $\mathrm{~T} 1$ & $0.54 \pm 0.59$ & $0.40 \pm 0.23$ & $16.6 \pm 7.20$ & $4.6 \pm 0.43$ & $17.8 \pm 0.1$ & $11.86 \pm 10.27$ \\
\hline & $\mathrm{T} 2$ & $0.01 \pm 0.02$ & $0.18 \pm 0.01$ & $100 \pm 0.01$ & $4.6 \pm 0.15$ & $17.8 \pm 0.1$ & 0 \\
\hline & T3 & $0.16 \pm 0.12$ & $0.25 \pm 0.20$ & $66.6 \pm 28.86$ & $4.3 \pm 0.40$ & $35.6 \pm 0.1$ & $5.93 \pm 10.27$ \\
\hline \multirow{3}{*}{4} & T1 & $0.45 \pm 0.56$ & $0.08 \pm 0.02$ & $20.83 \pm 7.20$ & $4.5 \pm 0.41$ & $17.8 \pm 17.8$ & $5.93 \pm 10.27$ \\
\hline & $\mathrm{T} 2$ & $0.30 \pm 0.03$ & $0.51 \pm 0.56$ & $54.2 \pm 43.90$ & $4.9 \pm 0.58$ & $11.86 \pm 10.27$ & $5.93 \pm 10.27$ \\
\hline & T3 & $0.60 \pm 0.52$ & $0.41 \pm 0.34$ & $441.6 \pm 14.43$ & $4.6 \pm 0.15$ & $23.73 \pm 10.27$ & $5.93 \pm 10.27$ \\
\hline \multirow{3}{*}{5} & $\mathrm{~T} 1$ & $0.65 \pm 0.36$ & $0.12 \pm 0.02$ & $25 \pm 0.01$ & $4.3 \pm 0.3$ & $11.8 \pm 10.27$ & $5.9 \pm 10.27$ \\
\hline & $\mathrm{T} 2$ & $0.09 \pm 0.04$ & $0.14 \pm 0.11$ & $83.3 \pm 28.86$ & $4.6 \pm 0.37$ & $29.7 \pm 10.27$ & $5.93 \pm 10.27$ \\
\hline & T3 & $0.11 \pm 0.10$ & $0.09 \pm 0.03$ & $83.3 \pm 28.86$ & $4.7 \pm 0.17$ & $23.73 \pm 10.27$ & 0 \\
\hline \multirow{3}{*}{6} & $\mathrm{~T} 1$ & $0.30 \pm 0.08$ & $0.03 \pm 0.08$ & $16.6 \pm 7.2$ & $4.6 \pm 0.32$ & $29.7 \pm 10.27$ & $5.93 \pm 10.27$ \\
\hline & $\mathrm{T} 2$ & $0.17 \pm 0.11$ & $0.10 \pm 0.01$ & $12.5 \pm 0.01$ & $4.7 \pm 0.36$ & $29.7 \pm 10.27$ & $5.93 \pm 10.27$ \\
\hline & T3 & $0.10 \pm 0.02$ & $0.07 \pm 0.04$ & $12.5 \pm 0.01$ & $4.6 \pm 0.15$ & $29.7 \pm 10.27$ & 0 \\
\hline \multirow{3}{*}{7} & $\mathrm{~T} 1$ & $0.09 \pm 0.17$ & $0.04 \pm 0.007$ & $12.5 \pm 0.01$ & $4.6 \pm 0.35$ & $47.46 \pm 10.27$ & $11.86 \pm 10.27$ \\
\hline & $\mathrm{T} 2$ & 0 & $0.06 \pm 0.02$ & $16.6 \pm 7.2$ & $4.6 \pm 0.25$ & $47.46 \pm 10.27$ & 0 \\
\hline & T3 & 0.25 & $0.08 \pm 0.03$ & $12.5 \pm 0.01$ & $4.6 \pm 0.1$ & $41.53 \pm 10.27$ & 0 \\
\hline \multirow{3}{*}{8} & $\mathrm{~T} 1$ & $0.14 \pm 0.01$ & $0.001 \pm 0.001$ & $12.5 \pm 0.01$ & $4.6 \pm 0.11$ & $47.46 \pm 10.27$ & $5.93 \pm 10.27$ \\
\hline & $\mathrm{T} 2$ & $0.12 \pm 0.04$ & $0.019 \pm 0.011$ & $20.83 \pm 7.2$ & $4.8 \pm 0.2$ & $41.53 \pm 10.27$ & 0 \\
\hline & T3 & $0.12 \pm 0.08$ & $0.041 \pm 0.026$ & $16.6 \pm 7.2$ & $4.6 \pm 0.15$ & $35.6 \pm 0.1$ & 0 \\
\hline \multirow{3}{*}{9} & $\mathrm{~T} 1$ & $0.16 \pm 0.01$ & 0 & $12,5 \pm 0.01$ & $4.8 \pm 0.1$ & $47.46 \pm 10.27$ & 0 \\
\hline & $\mathrm{T} 2$ & $0.17 \pm 0.02$ & $0.003 \pm 0.005$ & $25 \pm 0.1$ & $4.7 \pm 0.26$ & $59.3 \pm 10.27$ & 0 \\
\hline & T3 & $0.22 \pm 0.04$ & $0.020 \pm 0.010$ & $25 \pm 0.1$ & $4.6 \pm 0.15$ & $47.46 \pm 10.27$ & 0 \\
\hline \multirow{3}{*}{10} & $\mathrm{~T} 1$ & $0.09 \pm 0.03$ & $0.006 \pm 0.005$ & $25 \pm 0.1$ & $4.8 \pm 0.2$ & $29.6 \pm 10.27$ & 0 \\
\hline & $\mathrm{T} 2$ & $0.31 \pm 0.07$ & $0.010 \pm 0.004$ & $25 \pm 0.1$ & $4.7 \pm 0.15$ & $35.6 \pm 0.1$ & 0 \\
\hline & T3 & $1.28 \pm 0.31$ & $0.015 \pm 0.002$ & $25 \pm 0.1$ & $4.7 \pm 0,11$ & $41.53 \pm 1027$ & 0 \\
\hline \multirow{3}{*}{11} & $\mathrm{~T} 1$ & $0.54 \pm 0.41$ & $0.034 \pm 0.015$ & $12.5 \pm 0.01$ & $4.73 \pm 0.15$ & $23.73 \pm 10.27$ & 0 \\
\hline & $\mathrm{T} 2$ & $1.75 \pm 1.34$ & $0.030 \pm 0.008$ & $12.5 \pm 0.01$ & $4.6 \pm 0.05$ & $23.73 \pm 10.27$ & 0 \\
\hline & T3 & $1.70 \pm 0.37$ & $0.035 \pm 0.026$ & $54.16 \pm 43.89$ & $4.6 \pm 0.1$ & $29.6 \pm 10.27$ & 0 \\
\hline
\end{tabular}


Conociendo que la tasa de excreción de NAT es $0.0031 \mathrm{mg} / \mathrm{L} \mathrm{NAT/g}$ de pez/día se estima la tasa de excreción total de un sistema de $2.500 \mathrm{~L}$ con una biomasa de $30.000 \mathrm{~g}$, lo que es igual a $231 \mathrm{~g}$ NAT/día (0.0031 mg NAT/g/día x $2500 \mathrm{~L}=0.0077 \mathrm{~g} \mathrm{NAT/g/día} \times 30.000$ $\mathrm{g}$ de biomasa $=231 \mathrm{gNAT} /$ día). A partir de estos datos se calcula el área de biofiltración implementando la ecuación, el cual nos arrojó un área superficial de biofiltro de 1155m² (231 g NAT/día / 0.2 g NAT/ m²/día).

Los biofiltros además de mantener las concentraciones ideales de NAT para el crecimiento de los peces a través del proceso de nitrificación amonio-nitrito-nitrato, también generan el nitrato que es la fuente de nutriente para las plantas cultivadas en los sistemas acuapónicos (Timmons y Ebeling, 2007). Para determinar el número de plantas que pueden ser cultivadas en un sistema acuapónico es necesario conocer los requerimientos nutricionales de la especie cultivada y la tasa de nitrificación ó la tasa de conversión de NAT a nitrato/día, pues conociendo cuanto NAT excreta, cuanto de esté es transformado y la exigencia en Nitrógeno de la planta a cultivar, permite estimar cuantas plantas $/ \mathrm{m}^{2}$ puede mantener el sistema y así lograr una producción adecuada en plantas y peces.

Finalmente, los resultados obtenidos en este trabajo nos permite concluir que el nivel de proteína cruda tiene un efecto en el crecimiento de $C$, auratus, donde se obtuvo un mayor crecimiento en los individuos alimentados con el $45 \%$ de proteína cruda, y que la tasa de excreción de NAT está directamente relacionado con el incremento de nivel de proteína cruda, a mayor nivel de proteína cruda mayor excreción de NAT. Estos resultados son de gran importancia y utilidad para la producción en SCR y SA, ya que a partir de estos datos se puede estimar el tamaño de biofiltro y así estimar la carga máxima de biomasa/densidad de peces y a su vez el número de plantas que pueden ser mantenidos en los SA.

\section{AGRADECIMIENTOS}

A la vicerrectoría de investigaciones de la Universidad MilitarNueva Granadaporsuapoyo enel proyecto CIAS837.

\section{REFERENCIAS}

1. Abdo $\mathrm{Ml}$, Rodríguez-lbarra $\mathrm{E}$, Hernández C, Hernández K, González-Rodríguez B, Martínez-Rodríguez I y García-Ortega A. 2010. Efecto de diferentes niveles de proteína y lípidos totales en la dieta sobre el crecimiento y supervivencia de juveniles de pargo lunarejo Lutjanus guttatus. Revista de Biología Marina y Oceanografía, 45: 433-439.

2. Altinok I y Grizzle J. 2004. Excretion of ammonia and urea by phylogenetically diverse fish species in low salinities. Aquaculture, 238: 499-507.

3. Bandyopadhyay P, Swain SK y Mishra S. 2005. Growth and dietary utilization in goldfish (Carassius auratus Linn.) fed diets formulated with various local agro-produces. Bioresource Technology, 96: 731-740.

4. Ballestrazzi R, Lanari D y D'Agaro E. 1998. Performance, nutrient retention efficiency, total ammonia and reactive phosphorus excretion of growing European sea-bass (Dicentrarchus labrax, L.) as affected by diet processing and feeding level. Aquaculture, 161: 55-65.

5. Chakraborty SC y Chakraborty S. 1998. Effect or dietary protein level on excretion of ammonia in Indian major carp, Labeo rohita, fingerlings. Aquacult. Nutr, 4: 47-51.

6. Corraze G y Kaushik S. 2007. Nutrition and feeding of fish. Aquaculture, 267: 1-2.

7. Crear BJ y Forteath. 2002. Feeding has the largest effect on the ammonia excretion rate of the southern rock lobster, Jasus edwardsii, and western rock lobster, Panulirus Cygnus. Aquacultural Engineering, 26: 239-250.

8. Dinara-FAO. 2008. Estrategia general de la acuicultura sostenible de la República Orienta de Uruguay. Plan Nacional de Desarrollo de la Acuicultura- Uruguay, 68p.

9. Engin $\mathrm{K}$ y Carter CG. 2001. Ammonia and urea excretion rates of juvenile Australian 
short-finned eel (Anguilla australis australis) as influenced by dietary protein level. Aquaculture, 194: 123-136.

10. Gómez Y, Vargas J, Portavella M y López JC. 2006. Spatial learning and goldfish telencephalon NMDA receptors. Neurobiology of Learning and Memory, 85: 252-262.

11. Guan B, Hu W, Zhang T, Duan M, Li D, Wang Y y Zhu Z. 2010. Acute and chronic un-ionized ammonia toxicity to "all-fish" growth hormone transgenic common carp (Cyprinus carpio L.). Chinese Science Bulletin, 55: 4032-4036.

12. Guo Z, Zhu X, Liu J, Han D, Yang Y, Lan Z y Xie S. 2012. Effects of dietary protein level on growth performance, nitrogen and energy budget of juvenile hybrid sturgeon, Acipenser baerii $q \times$ A. gueldenstaedtii ${ }^{\lambda}$. Aquaculture, 338: 89-95.

13. Hanington PC, Barreda DR y Belosevic M. 2006. A Novel Hematopoietic Granulin Induces Proliferation of Goldfish (Carassius auratus L.) Macrophages. Journal of Biological Chemistry, 281: 9963-9970.

14. Ilmo D. 2009. Nutrición proteica y crecimiento en peces. Aspectos reguladores. Academias de ciencias matemáticas, Fisicoquímicas y Naturales de Granada, 76p.

15. Iregui $C$, Hernández $E$, Jiménez $A$, Pulido $A$, Lucía A, Comas J, Peña L y Rodríguez M. 2002. Manual de sanidad piscícola. Universidad Nacional de Colombia. Ministerio de agricultura y desarrollo rural. 62p.

16. Komiyama T, Kobayashi H, Tateno Y, Inoko H, Gojobori Ty lkeo K. 2009. An evolutionary origin and selection process of goldfish. Gene, 430: 5-11.

17. Leung KMY, Chu JCW y Wu RS. 1999. Effects of body weight, water temperature and ration size on ammonia excretion by the related grouper (Epinephelus areolatus) and mangrove snapper (Lutjanus argentimaculatus). Aquaculture, 170: 215-227.

18. Lim CB, Chew SF, Anderson PM y Ip YK. 2001. Reduction in the rates of protein and amino acid catabolism to slow down the accumulation of endogenous ammonia: a strategy potentially adopted by mudskippers (Periophthalmodon schlosseri and Boleophthalmus boddaerti) during aerial exposure in constant da. Journal of Experimental Biology, 204: 1605-1614.

19. Liu G, Yang S y Cheng H. 2009. Effect of temperature, stocking density and fish on the ammonia excretion in palmetto bass (Morone saxatilis $\times$ M. chrysops). Aquaculture research, 40: 450-455.

20. Losordo $T$ y Hobbs A. 2000. Using computer spreadsheets for water flow and biofilter sizing in recirculating aquaculture production systems. Aquacultural Engineering, 23:95-102.

21. Marshall O. 1995. Goldfish, a complete pet owner's manual. Borron's editorial. 2da.Ed. Hong Kong, 94p.

22. Martínez-Moreno O, Gómez-Ramírez E y HurtadoGiraldo H. 2011. Levante de Goldfish (Carassius auratus) en sistemas de recirculación cerrado. Revista Facultad de Ciencias Básicas, 7(2): 260-273

23. Mercado B, García J, Rosado R, Olaya C, Segura F, Bru S y Tordecilla G. 2006. Cultivo de dorada (Brycon Dahl, 1955) en jaulas flotantes a diferentes niveles de proteína. Revista Colombiana de Ciencias Pecuarias, v19: 204-211.

24. Merino E, Piedrahita RH y Conklin DE. 2007. Ammonia and urea excretion rates of California halibut (Paralichtys californicus, Ayres) under farm-like conditions. Aquaculture, 271: 227-243.

25. Moohammad H. 2009. Effect of dietary protein levels on growth performance and body composition of Nile tilapia, Orechromis niloticus L. Reared in Fertilizes Tanks. Departmet of Zoology. Pakistan Journal of Nutrition 8(5): 674-678.

26. Nerici C, Silva A y Merino G. 2012. Effect of two temperatures on ammonia excretion rates of Seriolella violacea (Palm fish) juveniles under rearing conditions. Aquacultural engineering, 46:47-52.

27. Randall DJ y Tsui TK. 2002. Ammonia toxicity in fish. Marine Pollution Bulletin, 45: 17-23.

28. Rakocy J. 2007. Design and operation of an aquaponics system. 1ra. Ed. Syaqua México. Panorama acuícola Magazine, 34p. 
29. Randall D. 2011. Excretion of Ammonia. p. 14371444. En: Farrell A (Ed). Encyclopedia of fish physiology: from genome to environment. 1ra. Ed. Vol: 1. Kate Mittell. Hong Kong. China, 2266p.

30. Richard W. 1980. Comparative physiology of animal sanenvironmentalapproach. EditorialReverte,901p.

31. Ruchimat $T$, Masumoto $T$, Mori $S$, Hosokawa $\mathrm{H}$ y Shimeno S. 1997. Effect of ration level on non-fecal nitrogen excretion of juvenile yellowtail (Seriola quinquradiata). Bulletin of Marine Science, 63:68.

32. Salazar MB y Ocampo D. 2002. Tasa de crecimiento del pez ángel Pterophyllum scalre (Perciformes: Chiclidae) en condiciones de laboratorio. Acta Universitaria, 12: 28-23.

33. Santosh Py Tibbetts S. 2009. Nutrition, feeding, and behavior of fish. Vet Clin Exot Animal, 12: 361-372.

34. Singh RK, Chavan SL, Desai AS y Khandagale PA. 2008. Influence of dietary protein levels and water temperature on growth, body composition and nutrient utilization of Cirrhinus mrigala (Hamilton, 1922) fry. Journal of Thermal Biology, 33: 20-26.

35. Sinha KA, Liew JH, Diricx M, Blust R y Boeck G. 2012. The interactive effects of ammonia exposure, nutritional status and exercise on metabolic and physiological responses in gold fish (Carassius auratus L). Aquatic Toxicology, 109: 33-46.

36. Timmons MB y Ebeling JM. 2007. Biofiltration. p. 242-.En: Timmons MB y Ebeling JM(Eds). Recirculating aquaculture. 2da. Ed. USDA, 489p.

37. Tulli F, Vachot C, Tibaldi E, Fournier $V$ y Kaushik SJ. 2007. Contribution of dietary arginine to nitrogen utilization and excretion in juvenile sea bass (Dicentrarchus labrax) fed diets differing in protein source. Comparative Biochemistry and Physiology Part A: Molecular \& Integrative Physiology, 147: 179-188.

38. Wajsbrot N, Gasith A, Diamant A, Popper DM. 1993. Chronic toxicity of ammonia to juvenile gilthead sea bream Sparus aurata and related histopathological effects. J. Fish Biol, 42: 321-328.
39. Webb KA y Gatlin DM. 2003. Effects of dietary protein level and form on production characteristics and ammonia excretion of red drum Sciaenops ocellatus. Aquaculture, 225:17-26.

40. WicksBJyRandallDJ.2002. The effect offeeding and fasting on ammonia toxicity in juvenile rainbow trout, Oncorhynchus mykiss. Aquatic toxicology, 59:71-82.

41. Wilkie PM. 1997. Mechanisms of ammonia excretion across fish gills. Comp. Biochem Physiol, Vol(1): 39-50.

42. Wilkie PM. 2002. Ammonia excretion and Urea Handling by Fish Gills: Present Understanding and Future Research Challenges. Journal of experimental zoology, 293:284-301.

43. Wood CM. 2006. Ammonia and urea metabolism andexcretion. p. 379-425. En: Evans Dy ClaiborneJB. The physiology of fishes. 3ra. Ed. Boca Raton, 616p.

44. Valbuena R y Vásquez W. 2011. Body weight is inversely associated with ammonia excretion in red tilapia (Oreochromis sp). Revista Colombiana de Ciencias Pecuaris, 24: 191-200.

45. Yang SD, Liou CH y Liu FG. 2002. Effects of dietary protein level on growth performance, carcass composition and ammonia excretion in juvenile silver perch (Bidyanus bidyanus). Aquaculture, 213 : 363-372.

46. Yuxiang W y Patrick JW. 2000. High ammonia tolerance in fishes of the family Batrachoididae (Toadfish and Midshipmen). Aquatic Toxicology, 50: 205-219.

47. Zhang A, Goodwin AE, Pfeijer TJ y Thomforde H. 2004. Effects of temperature and size on ammonia excretion by fasted golden shiners. North Am J Aquaculture, 66:15-19.

\section{CONSULTA PAGINAS WEB}

1. FAO. 2012. Estadística, Producción acuícola mundial. [http://faostat.fao.org/site/291/default.aspx. Consultada el 06 de Septiembre del 2013]. 